\title{
Estudo da Enxaqueca em Idade Pediátrica nos Cuidados de Saúde Primários da Região Centro de Portugal
}

\author{
Pediatric Migraine Study in Primary Health Care at Central Region of Portugal
}

\author{
Lopes Freitas $\mathrm{R}^{1, *}$, Mina $\mathrm{M}^{2}$, Madanelo $\mathrm{I}^{3}$, Silva $\mathrm{B}^{4}$, Silva $\mathrm{C}^{5}$, Cebola $\mathrm{J}^{6}$, Ribeiro $\mathrm{M}^{7}$, Tomás $\mathrm{L}^{8}$, Figueiredo $\mathrm{I}^{8}$, Amaral $\mathrm{L}^{9}$, \\ Paixão $L^{10}$, Esperança $A^{11}$, Pinto $V^{12}$, Rosendo I ${ }^{13}$, Palavra $F^{14}$ \\ 1-https://orcid.org/0000-0001-8980-5021 / Faculdade de Medicina da Universidade de Coimbra; Coimbra, Portugal \\ 2-Unidade de Saúde Familiar "A Ribeirinha"; Guarda, Portugal \\ 3-Unidade de Cuidados de Saúde Primários de Vouzela; Vouzela, Portugal \\ 4-Unidade de Saúde Familiar Pulsar; Coimbra, Portugal \\ 5-Unidade de Saúde Familiar Águeda+; Águeda, Portugal \\ 6-Unidade de Cuidados de Saúde Primários Fonte do Rei; Leiria, Portugal \\ 7-Unidade de Cuidados de Saúde Primários Figueira da Foz Urbana; Figueira da Foz, Portugal \\ 8-Unidade de Saúde Familiar Lusitana; Viseu, Portugal \\ 9-Unidade de Saúde Familiar Serra da Lousã; Lousã, Portugal \\ 10-Unidade de Saúde Familiar Coimbra Centro; Coimbra Portugal \\ 11-Unidade de Saúde Familiar Flor de Sal; Aveiro, Portugal \\ 12-Unidade de Cuidados de Saúde Primários Belmonte; Belmonte, Portugal \\ 13-Faculdade de Medicina da Universidade de Coimbra; Unidade de Saúde Familiar Coimbra Centro; Coimbra, Portugal \\ 14-https://orcid.org/0000-0002-2165-130X / Centro de Desenvolvimento da Criança - Neuropediatria, Hospital Pediátrico, Centro \\ Hospitalar e Universitário de Coimbra; Coimbra, Portugal
}

\section{Resumo}

Introdução: A enxaqueca é uma cefaleia primária comum, que pode ter início em idade pediátrica. Não existem dados concretos nos cuidados de saúde primários (CSP) em Portugal. Foi objetivo deste estudo caracterizar uma população pediátrica com diagnóstico de enxaqueca em seguimento nos CSP da região Centro de Portugal, abordando aspetos demográficos, epidemiológicos e clínicos.

Métodos: No primeiro trimestre de 2018, a partir de dados retrospetivos colhidos exclusivamente em ambiente de CSP, selecionámos uma amostra de indivíduos em idade pediátrica de 11 unidades de saúde, com diagnóstico de enxaqueca codificado nos problemas ativos do sistema informático. Foram recrutados sequencialmente e entrevistados de acordo com um guião pré-definido, baseado nos critérios da Classificação Internacional de Cefaleias. Realizámos a análise estatística descritiva das variáveis recolhidas.

Resultados: Recrutámos 117 indivíduos e, após entrevista, apenas 72 foram incluídos. Estimámos uma prevalência de enxaqueca pediátrica de 0,35\% nessas unidades, afetando predominantemente adolescentes do sexo feminino. $O$ intervalo de tempo decorrido entre o início dos sintomas e o diagnóstico teve uma mediana de 1,3 anos (0-3 anos). Confirmámos que faziam terapêutica profilática 22,2\% dos casos e terapêutica abortiva de crise 97,2\% deles, sendo as classes mais utilizadas os analgésicos simples $(65,7 \%)$ e anti-inflamatórios não esteróides (50,0\%). Apenas uma minoria utilizava triptanos (2,9\%). A automedicação foi reportada em 12,5\% dos casos. Recorriam à terapêutica não farmacológica 66,7\% dos doentes.

Discussão: $O$ diagnóstico e prevalência estimada de enxaqueca pediátrica nos CSP ficaram aquém dos valores descritos na literatura. $\bigcirc$ tratamento implementado nas crises é adequado, mas a utilização de terapêutica não farmacológica e profilática poderão ser otimizadas, nesta população particular. Salvaguarda-se, no entanto, que este foi um estudo preliminar de natureza retrospetiva realizado numa população de uma zona geo-gráfica específica do país, carecendo naturalmente de confirmação posterior.
Informações/Informations: Artigo Original, publicado em Sinapse, Volume 20, Número 1, janeiro-março 2020. Versão eletrónica em www.sinapse.pt Original Article, published in Sinapse, Volume 20, Number 1, january-march 2020. Electronic version in www.sinapse.pt (C) Autor (es) (ou seu (s) empregador (es)) 2020. Reutilização permitida de acordo com CC BY-NC. Nenhuma reutilização comercial.

(C) Author(s) (or their employer(s)) 2020. Re-use permitted under CC BY-NC. No commercial re-use.

\section{Palavras-chave:}

Cefaleia/diagnóstico; Cefaleia/tratamento farmacológico;

Criança;

Cuidados Primários de Saúde; Enxaqueca/diagnóstico;

Enxaqueca/tratamento farmacológico.

\footnotetext{
*Autor Correspondente / Corresponding Author: Elsa Rafaela Lopes Freitas Rua de Sanfalhos 674, Pedroso - 4415-330 Vila Nova de Gaia, Portugal rafaelalopes16@gmail.com

Recebido / Received: 2020-02-01 Aceite / Accepted: 2020-05-24 Publicado / Published: 2020-06-30

DOI: $10.46531 /$ sinapse/ AO/200007/2020
} 
Keywords:

Child;

Headache/diagnosis;

Headache/drug therapy;

Migraine Disorders/diagnosis;

Migraine Disorders/drug

therapy;

Primary Health Care.

\section{Abstract}

Introduction: Migraine is a common primary headache, which can begin in the pediatric age. There are no concrete data on primary health care $(\mathrm{PHC})$ in Portugal. This study aimed to characterize a pediatric population with migraine followed at $\mathrm{PHC}$ in the central region of Portugal, addressing demographic, epidemiological and clinical aspects.

Methods: In the first trimester of 2018, from retrospective data collected exclusively in a PHC environment, we selected a sample of pediatric individuals of 11 health units with a diagnosis of migraine encoded in the active problems of units' informatics system. They were sequentially recruited and interviewed according to a pre-defined guide, based on the International Classification of Headache Disorders criteria. A descriptive analysis of collected variables was conducted.

Results: We recruited 117 individuals and, after interview, only 72 were included. We estimated a prevalence of pediatric migraine of $0.35 \%$ in these units, affecting predominantly female adolescents. The time elapsed between the onset of symptoms and the diagnosis had a median of 1.3 years (0-3 years). We confirmed that prophylactic therapy was used by $22.2 \%$ of cases and abortive therapy by $97.2 \%$, with the most commonly used classes being simple analgesics (65.7\%) and non-steroidal anti-inflammatory drugs (50.0\%). A minority used triptans (2.9\%). Self-medication was reported in $12.5 \%$ of cases; $66.7 \%$ referred the usage of non-pharmacological therapy.

Discussion: The diagnosis and estimated prevalence of pediatric migraine in $\mathrm{PHC}$ units fell short of the values described in the literature. The abortive treatment was considered adequate, but the use of non-pharmacological and prophylactic therapies may be optimized in this particular population. However, this was a preliminary study of a retrospective nature carried out in a population of a specific geographic area of the country, naturally requiring further confirmation.

\section{Introdução}

A enxaqueca é uma cefaleia primária, comum e debilitante, não só pela dor, mas também pelos restantes sintomas associados. ${ }^{1,2}$ Pessoas de todas as idades podem referir queixas de cefaleia sugestivas de diagnóstico, incluindo crianças e adolescentes. ${ }^{2,3}$

De acordo com a bibliografia, estima-se que a prevalência da enxaqueca em idade pediátrica se encontre entre os $2,7 \%-10 \% .^{3,4}$ Tal traduz-se indubitavelmente numa diminuição da qualidade de vida de crianças e adolescentes e, possivelmente, do respetivo desempenho escolar. ${ }^{5-8} \mathrm{Em}$ Portugal, a literatura sobre a epidemiologia de enxaqueca em idade pediátrica é escassa. ${ }^{9}$

O diagnóstico da enxaqueca é clínico, baseando-se numa anamnese detalhada e num exame físico rigoroso. ${ }^{3,10}$ Os respetivos critérios de diagnóstico encontram-se elencados na Classificação Internacional de Cefaleias (ICHD), cuja versão 3 (definitiva) se publicou recentemente.' Não obstante, as crianças e adolescentes afetadas por esta doença têm dificuldade em descrever re- trospetivamente as características dos episódios de dor e, deste modo, tornam a anamnese muito dependente da informação cedida pelos pais e/ou representantes legais. ${ }^{2} 11$ Assim, apesar da melhoria e uniformização do conhecimento científico, torna-se um verdadeiro desafio clínico o diagnóstico preciso desta entidade, sobretudo na idade pediátrica. ${ }^{12}$

Mais ainda, a abordagem terapêutica destes doentes deve ser individualizada e interdisciplinar., ${ }^{4,11,13}$ Porém, a escolha da terapêutica analgésica e a eventual opção pela utilização de um agente profilático podem ser complexas. ${ }^{10}$ Apesar da maioria da população pediátrica ser eficazmente tratada nos cuidados de saúde primários (CSP)," crianças e adolescentes são frequentemente referenciados aos cuidados hospitalares, implicando a intervenção da Neurologia Pediátrica. ${ }^{6}$ Existe uma noção empírica relacionada com uma relutância genérica relativa à utilização de fármacos nesta população em particular, mas tal é também um aspeto que merece ser clarificado e desmistificado, para melhoria geral da qua- 
lidade de vida das crianças e dos adolescentes.

Em suma, na população pediátrica, a enxaqueca carece de investigação de natureza clínica, não só a nível diagnóstico, como também terapêutico. Este estudo teve como objetivo caracterizar uma amostra pediátrica de indivíduos com diagnóstico de enxaqueca, inscritos em unidades de CSP da região centro de Portugal, analisando variáveis demográficas, epidemiológicas e clínicas.

\section{Material e Métodos}

presente estudo observacional, retrospetivo, descritivo e multicêntrico, realizou-se durante o primeiro trimestre de 2018, em onze unidades prestadoras de CSP da região centro de Portugal, nomeadamente nos agrupamentos de centros de saúde (ACES) do Baixo Mondego, Baixo Vouga, Cova da Beira, Dão Lafões, Pinhal Interior, Pinhal Litoral e Unidade Local de Saúde da Guarda.

O estudo teve início após a aprovação da comissão de ética da Administração Regional de Saúde Centro. $\bigcirc$ consentimento informado foi assinado por todos os participantes e/ou respetivos representantes legais.

A população-alvo abrangeu os indivíduos em idade pediátrica (< 18 anos), com diagnóstico de enxaqueca (código N89) nos problemas ativos, recolhidos através das plataformas MIM@UF ou Medicine One, seguidos em consulta de medicina geral e familiar (MGF) nas onze unidades de saúde que participaram no estudo. A utilização das referidas plataformas, em cada uma das unidades de saúde, ocorria havia um período variável de tempo, mas em todos os casos superior a I ano. Determinou-se o número total de doentes com idade inferior a 18 anos de cada unidade de saúde participante, usando as mesmas plataformas informáticas, para se estimar a prevalência de enxaqueca em idade pediátrica nestas unidades.

Os indivíduos que cumpriam os critérios de inclusão foram convidados a participar no estudo por contacto telefónico. Posteriormente, à medida que compareciam sequencialmente à consulta de MGF, devidamente acompanhados dos representantes legais, participaram numa entrevista clínica estruturada, organizada e construída a partir dos critérios da ICHD-3beta (por, na altura, ser a versão vigente da ICHD) que incluía variáveis como: sexo, idade atual (em anos), idade de início dos sintomas (em anos), idade do diagnóstico (em anos), sintomatologia, existência de sintomas compatíveis com síndromes episódicos que podem estar associados a enxaqueca, referenciação para cuidados hospitalares (e respetiva idade), seguimento atual em cuidados hospitalares, terapêutica farmacológica profilática e abortiva da crise, quem a prescreveu e, por último, medidas não farmacológicas adotadas para o controlo da dor.

Esses elementos foram registados numa folha de cálculo de acordo com uma codificação composta pelas iniciais da unidade de saúde e pela ordem numérica do comparecimento na consulta, mantendo-se desta forma o anonimato do doente. Foram excluídos os indivíduos que, apesar de possuírem diagnóstico codificado nas plataformas informáticas, não compareceram à consulta de MGF e os que, após realização da entrevista clínica, se concluiu apresentarem outros diagnósticos que não o de enxaqueca.

O tratamento farmacológico foi ainda classificado como "Adequado" ou "Não adequado", de acordo com vários critérios: recomendações de painéis de peritos sobre a utilização de fármacos para tratamento da enxaqueca em idade pediátrica, ${ }^{14}$ informação contida no resumo das características do medicamento (RCM) relativamente à sua utilização em indivíduos com idade inferior a 18 anos e a dose prescrita avaliada à luz do que seria a dose-alvo para o percentil 50 (P50) do peso para a idade e sexo da criança ou adolescente, segundo as curvas do Centers for Disease Control and Prevention (CDC).

\section{I - Análise Estatística}

Toda a análise foi efetuada com recurso ao software IBM SPSS Statistics $23^{\circledR}$ para Windows ${ }^{\circledR}$.

As variáveis qualitativas foram descritas com recurso a frequências absolutas e relativas, enquanto que as quantitativas foram descritas pela sua média, desvio padrão, mínimo, mediana, máximo e primeiro e terceiro quartis. A normalidade das variáveis quantitativas foi avaliada recorrendo ao teste de Kolmogorov-Smirnov ou ao teste de Shapiro-Wilk, quando apropriado. Considerou-se estatisticamente significativa qualquer diferença em que $p<0,05$.

\section{Resultados}

Neste estudo, da amostra total dos 20832 indivíduos em idade pediátrica seguida no conjunto das II unidades de saúde, II 7 indivíduos apresentavam como problema ativo o diagnóstico de enxaqueca (codificado como N89), nas plataformas informáticas utilizadas. Cumpriam critérios de inclusão 72 desses indivíduos $(6 \mathrm{I}, 5 \%)$, estimando-se uma prevalência de 0,35\% de 
enxaqueca na população pediátrica em seguimento nas unidades de saúde que participaram no estudo (mínimo: 0,07\%; máximo: 2,99\%).

Foram excluídos 45 dos II 7 indivíduos inicialmente identificados (38,5\%), dado que 34 não compareceram à consulta de MGF e II, após a realização da entrevista clínica baseada nos critérios da ICHD-3beta, apresentavam outros diagnósticos (Fig.1).

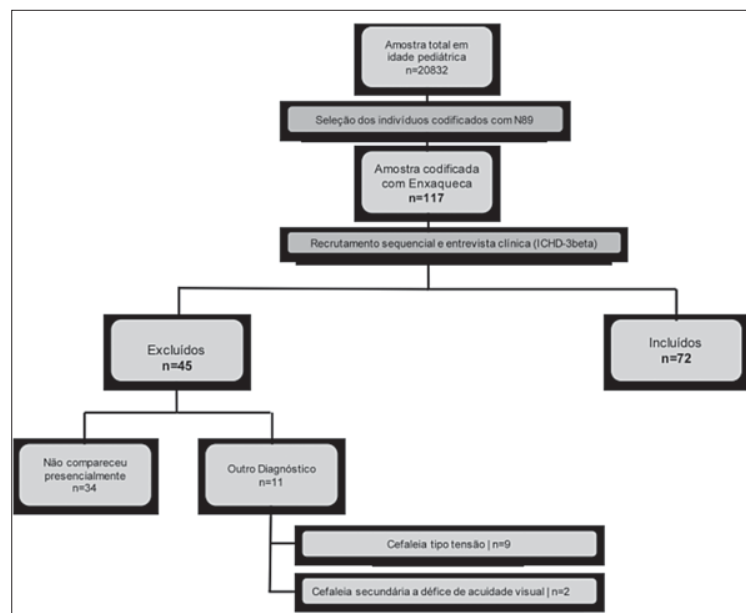

Figura 1. Fluxograma relativo ao método de seleção da amostra em estudo.

Os aspetos sociodemográficos, características clínicas e os dados referentes ao diagnóstico encontram-se apresentados nas Tabelas 1 e 2. A maioria dos indivíduos eram adolescentes ( $>10$ anos) $(86,1 \%)$ do sexo feminino $(58,3 \%)$. A idade mediana de início dos sintomas foi a de 8 anos (média de 8,3 \pm 3,6 anos) e o diagnóstico de enxaqueca foi feito mais tarde, com mediana de II anos (10,5 \pm 3,0 anos). O intervalo de tempo decorrido entre o início dos sintomas e a concretização do
Tabela 2. Características clínicas da enxaqueca na população incluída no estudo.

\begin{tabular}{|c|c|}
\hline & Frequência (\%) \\
\hline $\begin{array}{l}\text { Intensidade da dor } \\
\text { - Fraca } \\
\text { - Média } \\
\text { - Forte }\end{array}$ & $\begin{array}{l}0(0 \%) \\
28(38,9 \%) \\
44(61,1 \%)\end{array}$ \\
\hline $\begin{array}{l}\text { Localização } \\
\text { - Unilateral } \\
\text { - Bilateral } \\
\text { - Indefinida }\end{array}$ & $\begin{array}{l}10(13,9 \%) \\
46(63,9 \%) \\
16(22,2 \%)\end{array}$ \\
\hline Pulsatilidade & $44(61,1 \%)$ \\
\hline $\begin{array}{l}\text { Sintomas acompanhantes } \\
\text { - Náuseas } \\
\text { - Vómitos } \\
\text { - Fotofobia } \\
\text { - Fonofobia }\end{array}$ & $\begin{array}{l}51(70,8 \%) \\
35(48,6) \\
61(84,7 \%) \\
61(84,7 \%)\end{array}$ \\
\hline Fatores agravantes & $38(52,8 \%)$ \\
\hline $\begin{array}{l}\text { Pródromo ou aura } \\
\text { - Fadiga } \\
\text { - Palidez } \\
\text { - Dificuldade de concentração } \\
\text { - Alteração do comportamento } \\
\text { - Alodinia } \\
\text { - Sintomas visuais } \\
\text { - Défice motor de carácter focal } \\
\text { - Parestesias de carácter focal } \\
\text { - Disartria }\end{array}$ & $\begin{array}{l}58(80,6 \%) \\
35(48,6 \%) \\
29(40,3 \%) \\
22(30,6 \%) \\
12(16,7 \%) \\
2(2,8 \%) \\
26(36,1 \%) \\
13(18,1 \%) \\
6(8,3 \%) \\
6(8,3 \%)\end{array}$ \\
\hline $\begin{array}{l}\text { Equivalentes Migrainosos } \\
\text { - Perturbação gastrointestinal } \\
\text { recorrente } \\
\text { - vómitos cíclicos } \\
\text { - enxaqueca abdominal } \\
\text { - Vertigem paroxística benigna } \\
\text { - Torcicolo paroxístico benigno }\end{array}$ & $\begin{array}{l}23(31,9 \%) \\
7(9,7 \%) \\
15(20,8 \%) \\
11(15,3 \%) \\
3(4,2 \%)\end{array}$ \\
\hline
\end{tabular}

diagnóstico de enxaqueca teve uma mediana de I,3 anos (média de 2,2 $\pm 2,4$ anos).

No que diz respeito à sintomatologia, a maioria apresentava cefaleia de intensidade forte $(61,1 \%)$, bilateral $(63,9 \%)$ e pulsátil $(61,1 \%)$. A maioria tinha sintomas acompanhantes $(97,2 \%)$ e foram referidos fatores agravantes em $52,8 \%$ dos casos. A maioria referia algum tipo

Tabela 1. Características sociodemográficas e diagnósticas da população incluída no estudo.

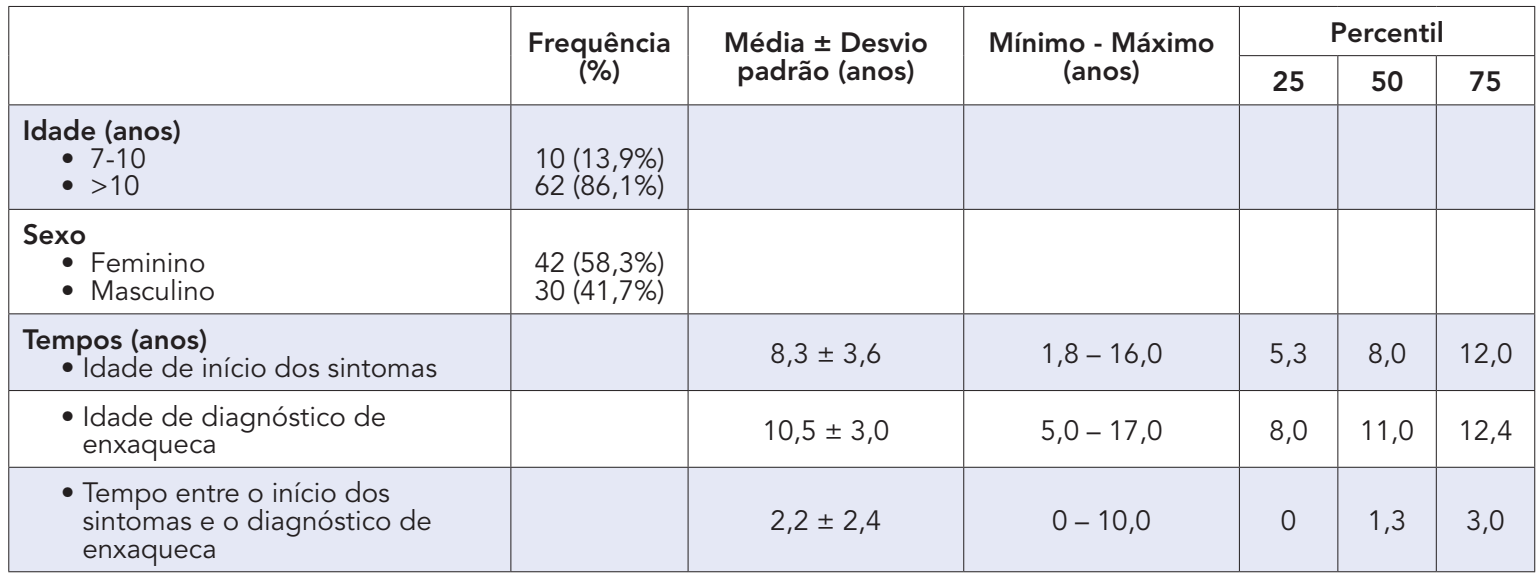


de sintoma compatível com pródromo (80,6\%). Apenas uma minoria reportava sintomatologia possivelmente compatível com síndromes episódicos que podem estar associados a enxaqueca (31,9\%). Frequentavam apenas a consulta de MGF $56,9 \%$ dos casos. Foram referenciados para consulta hospitalar $43,1 \%$ dos indivíduos e
45,2\% ainda permaneciam em controlo periódico, considerando a data de encerramento do estudo.

O recurso à terapêutica farmacológica foi uma atitude praticamente universal, na população estudada (98,6\%). Apenas 16 doentes (22,2\%) utilizavam terapêutica profilática (Tabela 3), dos quais 3 não sabiam

Tabela 3. Terapêutica farmacológica profilática na enxaqueca pediátrica, na amostra estudada.

\begin{tabular}{|c|c|c|c|c|c|}
\hline $\begin{array}{l}\text { Classe dos } \\
\text { fármacos }\end{array}$ & Fármaco & $\begin{array}{l}\text { Frequência } \\
\text { absoluta }\end{array}$ & $\begin{array}{l}\text { Adequação do } \\
\text { fármaco }\end{array}$ & Dose diária & $\begin{array}{c}\text { Adequação da } \\
\text { dose }\end{array}$ \\
\hline $\begin{array}{l}\text { Inibidor dos } \\
\text { canais de cálcio }\end{array}$ & Flunarizina & 5 & + & $5 \mathrm{mg}$ & + \\
\hline \multirow{2}{*}{ Antiepilético } & Carbamazepina & 1 & - & * & * \\
\hline & Topiramato & 3 & + & $25 \mathrm{mg}$ & + \\
\hline $\begin{array}{l}\text { Antidepressivo } \\
\text { tricíclico }\end{array}$ & Amitriptilina & 1 & + & $25 \mathrm{mg}$ & + \\
\hline Beta-bloqueador & Propranolol & 2 & + & $10 \mathrm{mg}$ & - \\
\hline Triptano & Frovatriptano & 1 & - & $2,5 \mathrm{mg}$ & - \\
\hline Procinético & Domperidona & 1 & - & $10 \mathrm{mg}$ & - \\
\hline Benzodiazepina & Bromazepam & 1 & - & $3 \mathrm{mg}$ & - \\
\hline
\end{tabular}

Nota: nas células referentes à adequação do fármaco e da dose: + adequado; - não adequado;

*caso não avaliado, por desconhecimento da posologia.

Tabela 4. Terapêutica farmacológica abortiva de crise utilizada na amostra estudada.

\begin{tabular}{|c|c|c|c|c|c|}
\hline $\begin{array}{l}\text { Classe dos } \\
\text { fármacos }\end{array}$ & Fármaco & $\begin{array}{l}\text { Frequência } \\
\text { absoluta }\end{array}$ & $\begin{array}{l}\text { Adequação do } \\
\text { fármaco }\end{array}$ & Posologia & $\begin{array}{c}\text { Adequação } \\
\text { da dose }\end{array}$ \\
\hline \multirow{2}{*}{ Analgésico } & Paracetamol & 45 & + & 10 a 15 mg/kg/dose & + \\
\hline & Metamizol & 1 & + & $\star$ & * \\
\hline \multirow{2}{*}{$\begin{array}{l}\text { Anti-inflamatório } \\
\text { não-esteróide }\end{array}$} & Ibuprofeno & 34 & + & 5 a $10 \mathrm{mg} / \mathrm{kg} /$ dose & + \\
\hline & Naproxeno & 1 & + & $250 \mathrm{mg}$ id & + \\
\hline $\begin{array}{l}\text { Relaxante } \\
\text { muscular }\end{array}$ & Tiocolquicósido & 1 & - & 4 mg id & - \\
\hline Triptano & Zolmitriptano & 2 & + & $2,5 \mathrm{mg}$ id & + \\
\hline Anti-histamínico & Dimenidrinato & 1 & + & $50 \mathrm{mg}$ id & + \\
\hline $\begin{array}{l}\text { Inibidor dos } \\
\text { canais de cálcio }\end{array}$ & Flunarizina & 1 & + & $5 \mathrm{mg}$ id & + \\
\hline
\end{tabular}

Nota: nas células referentes à adequação do fármaco e da dose: + adequado; - não adequado;

*caso não avaliado, por desconhecimento da posologia.

especificar os fármacos utilizados. Os doentes sob terapêutica profilática iniciaram-na, em média, aos $11,2 \pm$ 3,9 anos, sendo a idade mínima de 4 anos e máxima de 16 anos. Considerando os indivíduos que já interromperam a terapêutica farmacológica profilática, verificou-se que a realizaram durante I,8 \pm I,5 anos em média, sendo que o mínimo e máximo de anos de tratamento foi de 1 e 6 , respetivamente. Apenas 4 doentes mantinham esta terapêutica, à data do encerramento do estudo. A maioria dos indivíduos encontrava-se adequadamente tratada, quanto à classe farmacológica em uso $(69,2 \%)$ e à posologia escolhida (53,8\%). Dois indivíduos referiam a utilização de mais do que um fármaco profilático. A maioria iniciou este tipo de tratamento no contexto da consulta de Neurologia Pediátrica ( I I, I\%), seguindo-se a consulta de Pediatria (8,3\%) e a de MGF $(2,8 \%)$.

No que diz respeito à terapêutica abortiva da crise, 97,2\% dos indivíduos utilizavam-na. Destes, 46 indivíduos utilizavam analgésicos simples (65,7\%), 35 anti-inflamatórios não esteróides (AINE) (50\%) e dois triptanos (2,9\%). 
Encontravam-se medicados com mais do que um fármaco 15 indivíduos (2।,4\%). Estes doentes encontravam-se, na sua maioria $(92,9 \%)$, tratados de forma adequada quanto à classe farmacológica e posologia usada, de acordo com os critérios previamente explicitados. Apenas um indivíduo se encontrava inadequadamente tratado com um relaxante muscular (tiocolquicósido) para a crise de enxaqueca. Adicionalmente, quatro indivíduos não sabiam referir as doses utilizadas. A Tabela 4 resume esta informação. Os especialistas em MGF foram os que mais prescreveram este tipo de fármacos $(47,2 \%)$, seguidos do Pediatra $(25,0 \%)$ e do Neurologista (18,1\%). Nove indivíduos referiam automedicação (I2,5\%). Alguns indivíduos encontravam-se medicados e controlados por mais do que um especialista $(5,7 \%)$.

Relativamente à terapêutica não farmacológica, 48 indivíduos utilizavam-na $(66,7 \%)$, sendo que a maioria preferia o repouso $(56,9 \%)$ (inclui sono), escuridão $(38,9 \%)$ e o silêncio $(27,6 \%)$. O recurso a intervenções de natureza não farmacológica incluiu ainda as seguintes estratégias: aplicação de gelo (6,9\%); toalha de água fria na região frontal (6,9\%); restrição alimentar (2,8\%); café (2,8\%); pressionar a região dolorosa $(2,8 \%)$; exercícios de relaxamento ( $1,4 \%)$.

\section{Discussão}

Estudos epidemiológicos prévios referem que a enxaqueca está largamente subdiagnosticada e subtratada mundialmente, principalmente em crianças e adolescentes. ${ }^{15}$ De facto, tal como esperado, estimámos uma prevalência de enxaqueca pediátrica de $0,35 \%$ na zona geográfica servida pelas Unidades de Saúde participantes no estudo, que fica muito aquém do expectável, comparativamente com outros estudos, (embora se reforce que os dados não são, de todo, consensuais). ${ }^{9}$ Para isto podem concorrer, hipoteticamente, três causas imediatas: a subcodificação informática, a codificação equívoca do próprio diagnóstico (à falta de melhor epíteto, assume-se a enxaqueca como diagnóstico mais provável) ou o subdiagnóstico propriamente dito, não se identificando as características clínicas que, na criança e no adolescente, permitem estabelecer o diagnóstico da cefaleia primária em análise.

Também como descrito, a partir da adolescência ( $>10$ anos) a enxaqueca acomete sobretudo o sexo feminino. ${ }^{16,17}$ No entanto, na população estudada não encontrámos uma diferença tão acentuada entre sexos, tal como descrito por Lewis et al (I,3 vezes versus 3 vezes).

Quanto aos aspetos diagnósticos propriamente ditos, este é o primeiro estudo que investiga o tempo decorrido até ao diagnóstico de enxaqueca em idade pediátrica em Portugal, que seja do nosso conhecimento. Encontrou-se, de facto, um atraso no diagnóstico com uma mediana de I,3 anos, o que, apesar de tudo, é mais favorável do que o que se regista noutros países europeus, como a Itália. ${ }^{18}$ Este hiato poderá ser justificado pela pouca relevância que as crianças, os adolescentes e os próprios pais ou representantes legais tendem a atribuir às queixas de cefaleia, procurando ajuda médica apenas quando o impacto das crises se repercute na dinâmica familiar e escolar. No nosso estudo, foram encontrados $9,4 \%$ de indivíduos com um diagnóstico errado de enxaqueca, que se traduziria numa abordagem terapêutica provavelmente inapropriada e ineficaz. ${ }^{2}$ Este é um aspecto que reforça a importância (e necessidade) da formação contínua nesta área e da divulgação da formulação dos critérios de diagnóstico mais atual, para que todos os profissionais de saúde se sintam confortáveis, desde logo, na identificação e no diagnóstico dos variados tipos de cefaleia que podem surgir na criança e no adolescente.

Quanto aos aspetos terapêuticos, à semelhança do que está descrito na literatura, apenas uma minoria dos doentes fazia profilaxia. ${ }^{19}$ A realização de terapêutica profilática poderá ser um indicador de gravidade da enxaqueca e, efetivamente, a decisão de iniciar terapêutica neste sentido foi mais frequentemente assumida por Neurologistas Pediátricos. A flunarizina foi o fármaco mais comumente utilizado na prevenção da enxaqueca e, de facto, vários estudos comprovam a sua eficácia. ${ }^{20,21} \mathrm{O}$ propranolol, pouco estudado em crianças e adolescentes, ${ }^{3}$ quando utilizado, encontrava-se em doses infraterapêuticas, de acordo com a nossa análise dos registos de prescrição.

É importante realçar que, em alguns casos, o fármaco escolhido para profilaxia foi considerado desadequado. Relativamente ao frovatriptano, pode ser utilizado em ciclos curtos (de miniprofilaxia) na enxaqueca associada ao período menstrual, ${ }^{19,22}$ mas, no caso do nosso estudo, o indivíduo tratado dessa forma era do sexo masculino, o que exclui essa possibilidade terapêutica. Foram igualmente considerados desadequados a carbamazepina, a domperidona e o bromazepam, apesar de não se conhecerem os antecedentes pessoais dos indivíduos sob tais regimes terapêuticos, a ponto de se encontrar uma explicação plausível para a sua utilização prática.

Todos os indivíduos faziam terapêutica abortiva de crise, à exceção de dois, sobretudo prescrita pela especialidade de MGF, contrariamente ao que é descrito por Colombo 
et al. $\left.\right|^{8}$ Tal facto demonstra que os Médicos de Família das unidades de saúde que integraram o estudo se encontram confortáveis na gestão farmacológica desta situação clínica.

Tal como está descrito na literatura, verificámos que a terapêutica abortiva de crise mais utilizada consistiu em analgésicos simples e AINE, ${ }^{9,23}$ sendo que apenas uma pequena percentagem de indivíduos reportou $\mathrm{O}$ uso de triptanos. ${ }^{24}$ Todavia, de acordo com um artigo de evidência clínica baseado numa revisão sistemática da Cochrane, não há superioridade de nenhuma destas classes em relação aos triptanos, ${ }^{25,26}$ na população pediátrica.

Ressalva-se que quase todos os doentes se encontram adequadamente tratados na crise, registando-se uma gestão bastante eficiente desta situação clínica, nos cuidados de saúde primários. ${ }^{15}$ Relativamente aos analgésicos, a utilização de paracetamol na dose de 10 a $15 \mathrm{mg} / \mathrm{kg} /$ dose, ${ }^{25}$ em formulação oral ou retal, demonstrou ser uma abordagem bastante utilizada e eficaz, particularmente em concomitância com terapêutica não farmacológica, como o repouso. ${ }^{10}$ Todos os doentes tinham doses terapêuticas adequadas de acordo com o resumo das características do medicamento (RCM) e o P50 para o peso, de acordo com a idade e o sexo. No que diz respeito à utilização do metamizol, este é um fármaco útil no controlo da dor, ${ }^{21}$ mas raramente utilizado na prática ambulatória, particularmente em crianças e adolescentes. Ainda assim, o indivíduo que o referiu usar como abortivo da crise desconhecia a respetiva dose e frequência habitual de administração, pelo que não foi considerado na análise da adequação terapêutica. Relativamente aos AINE, o ibuprofeno foi o fármaco mais utilizado. De facto, é também um fármaco de primeira linha por ser relativamente seguro e efetivo. A posologia considerada adequada foi de 5 a 10 $\mathrm{mg} / \mathrm{kg} /$ dose, ${ }^{25}$ de acordo com o P50 do peso para a idade e o sexo e todos os indivíduos se encontravam medicados com doses adequadas. Quanto ao naproxeno, geralmente utilizado como segunda linha, considerou-se como dose adequada a de 2,5 a $5 \mathrm{mg} / \mathrm{kg} /$ dose, pelo que o indivíduo que o referiu utilizar se considerou também adequadamente tratado. Dois indivíduos referiam utilizar zolmitriptano oral na crise de enxaqueca. Este é um fármaco com um bom perfil de segurança ${ }^{27}$ em adolescentes, mas a informação sobre a eficácia é muito variável..$^{28}$ Como posologia adequada consideraram-se 2,5 a $5 \mathrm{mg}$ por toma, ${ }^{10}$ pelo que um dos indivíduos se encontrava tratado de forma adequada. No entanto, um outro não soube especificar a dose habitual com que se encontrava medicado.

O dimenidrinato funciona como um antiemético eficaz no tratamento sintomático de náuseas e vómitos. Este deve ser utilizado na dose de 50 a 100 mg oralmente, em crianças com idades superiores ou iguais a 12 anos, pelo que a sua utilização se considerou apropriada, nos casos em que tal foi referido, ${ }^{21}$ essencialmente como adjuvante analgésico na crise de enxaqueca. A flunarizina é um inibidor dos canais de cálcio utilizado como terapêutica profilática. Havia apenas um indivíduo a fazer este fármaco na dose de $5 \mathrm{mg}$ e num regime que sugeria a sua utilização como abortivo da crise de enxaqueca. Ainda que a utilização deste medicamento possa fazer sentido, neste contexto (considerando-se adequada), a flunarizina não deve ser usada como fármaco abortivo da crise. O tiocolquicósido é um relaxante muscular utilizado em adolescentes com mais de 16 anos como tratamento adjuvante para contraturas musculares dolorosas. Sendo que se trata de um fármaco que não tem indicação na profilaxia da enxaqueca, considerámos a sua utilização desadequada.

Ainda assim, é de realçar a percentagem considerável de automedicação que foi encontrada. Tal não parece necessariamente negativo, dado poder traduzir uma capacitação efetiva das famílias para a gestão da crise de enxaqueca, sem haver necessidade da constante aprovação médica para a manipulação de analgésicos simples e AINE (pois foram efetivamente estes os fármacos mais usados). No extremo oposto encontram-se as famílias em que a criança, o adolescente ou o representante legal não sabiam referir a terapêutica habitual. Nestes casos, o grau de capacitação para a gestão da terapêutica será necessariamente mais baixo, reforçando o trabalho que, a este nível, poderá ser feito, por parte dos próprios profissionais de saúde.

Relativamente à terapêutica não farmacológica, à semelhança de outros estudos, o repouso (incluindo períodos de sono) foi utilizado pela maioria das crianças e adolescentes com enxaqueca. ${ }^{23}$ Contudo, será importante reforçar, junto das famílias e da própria comunidade escolar (dado que tal poderá implicar uma certa interferência no desempenho escolar da criança ou do adolescente), o benefício desta intervenção terapêutica inócua e simples, mas eficaz. De facto, em ambiente escolar, a existência de condições para que a criança possa repousar ou dormir num local com pouca luminosidade e silencioso pode ser uma estratégia muito útil na gestão desta situação.

\section{Conclusão}

Apesar de os critérios de diagnóstico estarem perfeitamente estabelecidos, a identificação assertiva de uma 
enxaqueca, em idade pediátrica, ainda se acompanha de um atraso significativo entre o início dos sintomas e o diagnóstico efetivo. Este é um aspecto que merece naturalmente um reforço junto dos profissionais de saúde, mas também da própria população em geral, com vista a combater um certo niilismo perante queixas de cefaleia em crianças e adolescentes. Esta é uma área que carece de investigação futura dirigida.

\section{Responsabilidades Éticas}

Conflitos de Interesse: Os autores declaram a inexistência de conflitos de interesse na realização do presente trabalho.

Fontes de Financiamento: Não existiram fontes externas de financiamento para a realização deste artigo.

Confidencialidade dos Dados: Os autores declaram ter seguido os protocolos da sua instituição acerca da publicação dos dados de doentes.

Proteção de Pessoas e Animais: Os autores declaram que os procedimentos seguidos estavam de acordo com os regulamentos estabelecidos pelos responsáveis da Comissão de Investigação Clínica e Ética e de acordo com a Declaração de Helsínquia da Associação Médica Mundial.

Proveniência e Revisão por Pares: Não comissionado; revisão externa por pares.

\section{Ethical Disclosures}

Conflicts of interest: The authors have no conflicts of interest to declare.

Financing Support: This work has not received any contribution, grant or scholarship

Confidentiality of Data: The authors declare that they have followed the protocols of their work center on the publication of data from patients.

Protection of Human and Animal Subjects: The authors declare that the procedures followed were in accordance with the regulations of the relevant clinical research ethics committee and with those of the Code of Ethics of the World Medical Association (Declaration of Helsinki).

Provenance and Peer Review: Not commissioned; externally peer reviewed.

\section{References / Referências}

1. Headache Classification Committee of the International Headache Society (IHS). The International Classification of Headache Disorders, 3rd edition. Cephalalgia. 2018;:1-211. doi: $10.1177 / 0333102417738202$.

2. Abu-Arafeh $A$, Andrasik F, Bellini $B$, Cescut $A, C r a b a F$, et al. Childhood headache - Clinics in Developmental Medicine. 2nd ed. London: Mac Keith Press; 2013.

3. Lewis D, Berkowitz C, Galloway G. Headache in children and adolescents - First Consult. ClinicalKey. 2011. [acedido Nov 2019] Disponível em: https://www.clinicalkey.com/

4. Damen L, Bruijn JK, Verhagen AP, Berger MY, Passchier $J$, Koes BW. Symptomatic treatment of migraine in children: a systematic review of medication trials. Pediatrics. 2005;116:e295-302.

5. Wöber-Bingol C. Epidemiology of migraine and headache in children and adolescents. Curr Pain Headache Rep. 2013;17:341. doi: 10.1007/s11916-013-0341-z.

6. Benzon H, Rathmell J, Turk D, Argoff C, Hurley R. Pediatric Chronic Pain Management. Practical Management of Pain. 5th ed. Amsterdam: Elsevier; 2013.

7. Hickman C, Lewis K, Little R, Rastogi R, Yonker M. Prevention for Pediatric and Adolescent Migraine. Headache. 2015;55:1371-81

8. Johnson A, Bickel J, Lebel A. Pediatric migraine prescrip- tion patterns at a large academic hospital. Ped Neurol. 2014;51:706-12. doi: 10.1016/j.pediatrneurol.2014.06.018.

9. Martins I. Enxaqueca - da Clínica para a Etiopatogenia. Acta Med Port. 2009;22:589-98.

10. Rubin D, Kornblau D, Conway E, Caplen S. Neurologic Disorders. Rosen's Emergency Medicine - concepts and clinical pratice. 8th ed. Amsterdam: Elsevier; 2014.

11. Harding A, Clark L. Pediatric migraine: common, yet treatable. Nurse Pract. 2014; 39:22-31. doi: 10.1097/01. NPR.0000454980.88918.f0.

12. Winner P, Hershey AD. Epidemiology and diagnosis of migraine in children. Curr Pain Headache Rep. 2007;11:375-82.

13. Faedda N, Cerutti R, Verdecchia P, Migliorini D, Arruda M, Guidetti V. Behavioral management of headache in children and adolescents. J Headache Pain. 2016;17:80.

14. Winner P. Pediatric and adolescent migraine. In: Information for health care professionals. Chicago: American Headache Society; 2009.

15. Stovner L, Hagen $K$, Jensen R, Katsarava Z, Lipton R, Scher $A$, et al. The global burden of headache: a documentation of headache prevalence and disability worldwide. CephalaIgia. 2007; 27:193-210.

16. Cvetkovic V, Plavec D, Lovrencic-Huzjan A, Strineka M, Azman D, Bene R. Prevalence and clinical characteristics of headache in adolescents: a Croatian epidemiological study. Cephalalgia. 2014; 34:289-97. doi: 10.1177/0333102413507636.

17. Lewis D. Headaches in Infants and Children. Swaiman's Pediatric Neurology - Principles and Practice. 5th ed. Amsterdam: Elsevier; 2012.

18. Colombo B, Dalla Libera D, De Feo D, Pavan G, Annovazzi PO, Comi G. Delayed diagnosis in pediatric headache: an outpatient Italian survey. Headache. 2011;51:1267-73. doi: 10.1111/j.1526-4610.2011.01976.x.

19. Silberstein S, Holland S, Freitag F, Dodick DW, Argoff C, Ashman E. Evidence-based guideline update: pharmacologic treatment for episodic migraine prevention in adults: report of the Quality Standards Subcommittee of the American Academy of Neurology and the American Headache Society. Neurology. 2012;78:1337-45. doi: 10.1212/ WNL.0b013e3182535d20.

20. Tajti J, Szok D, Csati A, Vecsei L. Prophylactic Drug Treatment of Migraine in Children and Adolescents: An Update. Curr Pain Headache Rep. 2016;20:1. doi: 10.1007/s11916015-0536-6.

21. Evers S, Afra J, Frese A, Goadsby PJ, Linde M, May A, et al. EFNS guideline on the drug treatment of migraine-revised report of an EFNS task force. Eur J Neurol. 2009;16:968-81. doi: 10.1111/j.1468-1331.2009.02748.x.

22. Macone $A E$, Perloff MD. Triptans and migraine: advances in use, administration, formulation, and development. Expert Opin Pharmacother. 2017:18:387-97. doi: 10.1080/14656566.2017.1288721.

23. Cavestro $C$, Montrucchio F, Benci $P$, Pompilio D, Mandrino $S$, Cencio PG, et al. Headache prevalence and related symptoms, family history, and treatment habits in a representative population of children in Alba, Italy. Ped Neurol. 2014;51:348-53. doi: 10.1016/j.pediatrneurol.2014.05.022.

24. Gallelli L, lannacchero $R$, De Caro $E$, Peltrone F, Colosimo M, De Sarro G. A questionnaire-based study on prevalence and treatment of headache in young children. J Headache Pain. 2005;6:277-80.

25. Faber AJ, Lagman-Bartolome A, Rajapakse T. Drugs for the acute treatment of migraine in children and adolescents. Paediatr Child Health. 2017;22:454-8. doi: 10.1093/pch/pxx170.

26. Richer L, Billinghurst $L$, Linsdell MA, Russell $K$, Vandermeer B, Crumley ET, et al. Drugs for the acute treatment of migraine in children and adolescents. Cochrane Database Syst Rev. 2016;4:CD005220. doi: 10.1002/14651858. CD005220.pub2.

27. Kacperski J, Hershey AD. Newly approved agents for the treatment and prevention of pediatric migraine. CNS Drugs. 2016;30:837-44. doi: 10.1007/s40263-016-0375-y.

28. McKeage K. Zolmitriptan nasal spray: a review in acute migraine in pediatric patients 12 years of age or older. Paediatr Drugs. 2016;18:75-81. doi: 10.1007/s40272-015-0160-2. 